\title{
Nouvelles précisions sur la carte génétique du système de groupes sanguins $B$ des bovins
}

\author{
F. GROSCLAUDE, J. LEFEBVRE * et G. NOE \\ I.N.R.A., Laboratoire de Génétique biochimique \\ * Laboratoire de Génétique factorielle, $F 78350$ Jouy-en-Josas
}

\begin{abstract}
Résumé
Onze nouveaux cas de recombinaison au sein du système de groupes sanguins $B$ ont été observés dans les races bovines Charolaise, Normande, Montbéliarde et Française Frisonne Pie-Noire. Par rapport aux conclusions précédentes (Grosclaude et al., 1979), l'analyse de ces cas permet de localiser avec plus de précision, dans la carte du système, les déterminants génétiques des facteurs $P_{1}, P_{2}, O_{1}, E_{1}^{\prime}, P^{\prime}{ }_{2}, F 20, B_{2} K$ et celui du facteur $E_{3}^{\prime}$ voisin de $T$. Les déterminants des facteurs $A^{\prime \prime}$ et $B^{\prime \prime}$ ont été introduits dans la carte. Il est vérifié que les facteurs $\mathrm{E}_{1}^{\prime}$ et $\mathrm{E}_{2}^{\prime}$ sont sous le contrôle de déterminants génétiques distincts. Par ailleurs, dans l'un des cas, on observe à nouveau l'absence du groupe $\mathrm{E}_{3}^{\prime} \mathrm{G}^{\prime \prime}$ dans un phénogroupe recombiné, pourtant issu d'un génotype homozygote pour ce groupe, ce qui conduit à admettre l'existence d'au moins deux sites non contigus pour $\mathrm{E}_{3}^{\prime} \mathrm{G}^{\prime \prime}$.

La relation entre le taux apparent de recombinaison intra-race au système $B$ et le taux de recombinaison entre les bornes du système fait l'objet d'une analyse préliminaire.
\end{abstract}

Mots-clés : Groupes sanguins, bovins, système $B$, recombinaison, carte génétique.

\section{Summary}

Additional data on the genetic map of the B system of cattle blood groups

Eleven additional cases of recombination within the B blood group system have been. observed in the Charolais, Normande, Montbéliarde and French Friesian cattle breeds. As compared to previous conclusions (GroscLAUDE et al., 1979), examination of those cases made possible a more precise localisation in the map of the system, of the genetic determinants of factors $P_{1}, P_{2}, O_{1}, E_{1}^{\prime}, P_{2}^{\prime}, F 20, B_{2} K$ and of the $E_{3}^{\prime}$ factor close to $T$. The determinants of factors $\mathrm{A}^{\prime \prime}$ and $\mathrm{B}^{\prime \prime}$ have been introduced in the map. Evidence is presented for the factors $E_{1}^{\prime}$ and $E_{2}^{\prime}$ having distinct genetic determinants. In addition, in one of the cases, the absence of the group $\mathrm{E}_{3}^{\prime} \mathrm{G}^{\prime \prime}$ in the recombined phenogroup derived from a genotype homozygous for this group was observed again; this led to the postulate that there are at least two sites for $\mathrm{E}_{3}^{\prime} \mathrm{G}^{\prime \prime}$.

The relation between the apparent within breed rate of recombination of the B system, and the rate of recombination between the limits of the system was preliminarily investigated.

Key-words : Blood groups, cattle, B system, recombination, genetic map. 


\section{Introduction}

Le système B, l'un des 11 systèmes de groupes sanguins connus chez les bovins, découvert par Stormont et al. (1951), contrôle à lui seul plus de la moitié des quelque 90 facteurs antigéniques identifiés dans cette espèce à l'aide des réactifs monospécifiques mis au point par les diverses équipes de recherche spécialisées dans le monde. Dans leur travail original, Stormont et al. (1951) ont montré que la plupart des allèles de ce système contrôlaient non pas un seul facteur antigénique, mais un groupe apparemment indissociable de facteurs antigéniques appelé «phénogroupe ( (STORMONT, 1955). Parmi les interprétations qui pouvaient être avancées pour expliquer ce phénomène, SToRMONT et al. (1951) avaient donné la préférence à l'idée que «les diverses spécificités du système $B$ pouvaient ne représenter que les propriétés sérologiques croisées d'une série d'antigènes étroitement apparentés, contrôlés par les allèles d'un seul gène ».

Toutefois, après la mise en évidence d'un premier cas par RENDEL (1958), les observations sur la transmission de phénogroupes remaniés, qui paraissent résulter de crossing-over simples au sein du système B, se multiplient (voir bibliographie complète dans Grosclaude et al., 1979). En 1966, Green, dans le Laboratoire de Stormont, propose une première ébauche de carte génétique du système, mais ce sont Bouw et ses collaborateurs (Bouw \& Frorentini, 1970 ; Oosterlee \& Bouw, 1974 ; RuiterKAMP et al., 1977) qui seront les premiers à entreprendre une étude systématique sur ce sujet. Dans la dernière version de la carte génétique proposée par cette équipe, RUITERKAMP et al. (1977) donnent la séquence des déterminants génétiques de 12 facteurs ou groupes de facteurs $\left(\mathrm{Q}-\mathrm{Y}_{2}-\mathrm{D}^{\prime}-\mathrm{G}^{\prime \prime}-\mathrm{F}^{\prime}-\mathrm{E}^{\prime}{ }_{1} \cdot \mathrm{Q}^{\prime}-\mathrm{I}_{2}-\mathrm{OK}^{\prime}-\mathrm{J}_{1}^{\prime} \mathrm{O}^{\prime} \mathrm{NF} 7-\mathrm{I}_{1}-\mathrm{I}^{\prime}\right)$; en outre, ces auteurs indiquent les intervalles dans lesquels se situent 13 autres déterminants. En matière d'interprétation génétique, RuITERKAMP et al. concluent que le polymorphisme du système $B$ est contrôlé non pas par un seul gène, mais par une série de gènes étroitement liés.

Nous avons, dans une publication précédente (Grosclaude et al., 1979), proposé une carte génétique du système $B$ plus élaborée que celle de Ruiterkamp et al. (1977), mais concordant assez bien, pour l'essentiel, avec cette dernière. Cette carte a été déduite des observations faites dans 40 familles (père, mère, produit) chez lesquelles l'un des phénogroupes reçus par le produit résultait, selon toute vraisemblance, d'un crossingover simple, survenu dans le système $B$ de l'un de ses parents. Des éléments complémentaires ont par ailleurs été déduits de rapprochements faits sur les caractéristiques de phénogroupes existant dans les races françaises. Nous avons, dans la même publication, estimé à 0,7 centimorgan la longueur opérationnelle du segment chromosomique correspondant au système $\mathbf{B}$, valeur qui nous a paru exclure l'hypothèse d'un déterminisme monogénique, et montré par ailleurs que des événements génétiques autres que le crossing-over simple (double crossing-over, délétion ou conversion génique) pouvaient affecter ce segment.

Le présent travail est consacré à l'analyse de 11 nouveaux cas de recombinaison, qui permet d'affiner la carte génétique du système $B$; les relations entre le taux apparent de recombinaison intra-race et le taux de recombinaison entre les bornes du système (longueur opérationnelle du segment chromosomique correspondant) font également l'objet d'une analyse préliminaire. 


\section{Matériel et méthodes}

Les 11 cas de recombinaison analysés dans ce travail ont été observés parmi les quelque 30000 familles (père, mère, produit) dont le groupe sanguin a été examiné depuis la rédaction de notre précédente publication (début 1979) par le Service d'analyse des groupes sanguins bovins de l'I.N.R.A., à Jouy-en-Josas, mais ils ne représentent qu'une partie des cas de recombinaison survenus dans ces familles. En effet tous les cas de recombinaison supposés n'ont pu faire l'objet des vérifications sérologiques ou génétiques indispensables. D'autre part, les cas de recombinaison équivalant à des répétitions de cas déjà répertoriés n'ont évidemment pas été retenus ici : à titre d'exemple, le cas $\mathrm{n}^{\circ} 24$ (Grosclaude et al., 1979) a désormais été observé 3 fois, et le cas $\mathrm{n}^{\circ} 25,2$ fois, parmi les 193 filiations informatives du même taureau.

Les techniques et la liste des réactifs utilisés sont les mêmes que dans Grosclaude et al. (1979). La nomenclature n'a évolué que pour le facteur précédemment, et provisoirement, appelé F16, qui a pris l'appellation définitive de $\mathrm{J}^{\prime \prime}$ (XIX ${ }^{e}$ Congrès de la Société I.S.A.B.R., Ottawa, 1982, non publié).

Les calculs relatifs à l'estimation du taux de recombinaison apparent intra-race ont été effectués sur micro-ordinateur Wang 2200 VP à l'aide d'un programme écrit à cet effet, en langage Basic.

Il paraît indispensable pour faciliter la compréhension du présent travail, de rappeler plusieurs définitions :

- réactif monospécifique : un antisérum qui ne peut plus être fractionné par absorption ;

- facteur antigénique : le caractère antigénique détecté par un réactif monospécifique ;

- sous-groupes linéaires : deux facteurs forment un système de sous-groupes linéaires lorsque l'un d'entre eux n'a jamais été observé sans l'autre (exp. : $Y_{1}$ et $Y_{2}$ ) ; il existe, dans le système $\mathbf{B}$, des systèmes de sous-groupes linéaires comportant 3 , ou 4 , facteurs antigéniques $\left(\mathrm{G}_{1}, \mathrm{G}_{2}, \mathrm{G}_{3} ; \mathrm{O}_{1}, \mathrm{O}_{2}, \mathrm{O}_{3}, \mathrm{O}_{\mathrm{x}} ; \mathrm{E}_{1}^{\prime}, \mathrm{E}_{2}^{\prime}, \mathrm{E}_{3}^{\prime}, \mathrm{E}_{4}^{\prime}\right)$;

- déterminant génétique : une séquence d'ADN, de longueur inconnue, codant une subdivision de la carte du système (un ou plusieurs facteurs), identifiable par crossingover.

Toutes ces définitions sont purement opérationnelles et n'ont aucune connotation explicative. La définition de réactif monospécifique, en particulier, mal comprise par certains auteurs (CARENZI et al., 1972) n'implique rien, a priori, ni sur les caractéristiques immunochimiques du facteur antigénique correspondant, ni sur ses relations avec d'autres facteurs (y compris sous la forme de sous-groupes), ni sur son déterminisme génétique, en particulier sur le nombre de déterminants génétiques codant pour ce facteur dans la séquence d'ADN du système considéré.

\section{Résultats}

\section{A. Analyse de 11 nouveaux cas de recombinaison}

Le tableau 1 récapitule les éléments de l'analyse de 11 cas de recombinaison informatifs observés dans le système $\mathrm{B}$ postérieurement à notre précédente publication 
(Grosclaude et al., 1979). Des déductions utiles à l'établissement de la carte génétique de ce système peuvent être faites à partir de chacun de ces cas, en supposant que le phénogroupe remanié résulte d'un crossing-over méiotique simple, et que ce crossingover n'a provoqué la disparition d'aucun facteur antigénique. Dans le cas $n^{\circ} 41$ par exemple, on voit facilement que les déterminants génétiques des facteurs $G_{2}, Y, D^{\prime}$, $\mathrm{G}^{\prime}$ et $\mathrm{Y}^{\prime}$ sont situés d'un côté du site du crossing-over, et ceux des facteurs $P_{1}, A^{\prime}, B^{\prime}$, $E^{\prime}, Q^{\prime}, B^{\prime \prime}$ et $I^{\prime \prime}$ de l'autre côté.

\section{TABLEAU 1}

Liste des cas de recombinaison analysés dans le présent travail.

List of the cases of recombination analysed in the present work.

\begin{tabular}{|c|c|c|c|c|}
\hline $\begin{array}{l}\text { Cas } \\
\mathrm{N}^{\circ}\end{array}$ & $\begin{array}{l}\text { Sexe (1) } \\
\text { parental }\end{array}$ & Phénogroupes parentaux (1) & $\begin{array}{l}\text { Phénogroupes } \\
\text { recombinés ( } 2)\end{array}$ & $\begin{array}{l}\text { Trans- } \\
\text { mission } \\
\text { (3) }\end{array}$ \\
\hline \multicolumn{5}{|c|}{ Charolaise } \\
\hline $\begin{array}{l}41 \\
42 \\
43 \\
44 \\
45 \\
46 \\
47\end{array}$ & $\begin{array}{l}\mathrm{M} \\
\mathrm{F} \\
\mathrm{M} \\
\mathrm{F} \\
\mathrm{M} \\
\mathrm{M} \\
\mathrm{M}\end{array}$ & 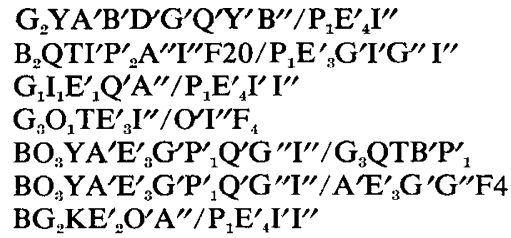 & $\begin{array}{l}\mathbf{G}_{2} \mathbf{P}_{1} \mathbf{Y} \mathbf{D}^{\prime} \mathbf{E}_{4}^{\prime} \mathbf{G}^{\prime} \mathbf{Y}^{\prime} \mathbf{I}^{\prime \prime} \\
\mathbf{B T} \mathbf{E}_{3}^{\prime} \mathbf{G}^{\prime} \mathbf{I}^{\prime} \mathbf{P}_{2}^{\prime} \mathbf{A}^{\prime \prime} \mathbf{G}^{\prime \prime} \mathbf{I}^{\prime \prime} \mathbf{F} 20 \\
\mathbf{I}_{1} \mathbf{Q}^{\prime} \\
\mathbf{O}_{1} \mathbf{I}^{\prime \prime} \\
\mathbf{O}_{3} \mathbf{Q} \mathbf{A}^{\prime} \mathbf{P}_{1}^{\prime} \mathbf{Q}^{\prime} \mathbf{I}^{\prime \prime} \\
\mathbf{A}^{\prime} \mathbf{G}^{\prime} \mathbf{F} 4 \\
\mathbf{B G}_{2} \mathbf{K} \mathbf{P}_{1} \mathbf{E}_{2}^{\prime} \mathbf{O}^{\prime}\left(\mathbf{A}^{\prime \prime}\right)\end{array}$ & $\begin{array}{l}- \\
+ \\
- \\
+ \\
+\end{array}$ \\
\hline \multicolumn{5}{|c|}{ Montbéliarde } \\
\hline $\begin{array}{l}48 \\
49\end{array}$ & $\begin{array}{l}\mathbf{M} \\
\mathbf{M}\end{array}$ & $\begin{array}{l}\mathrm{G}_{3} \mathrm{O}_{1} \mathrm{TE}_{3}^{\prime} \mathrm{K}^{\prime} / \mathrm{YE}_{3}^{\prime} \mathrm{G}^{\prime} \mathrm{I}^{\prime} \mathrm{G}^{\prime \prime} \\
\mathrm{P}_{2 .} \mathrm{E}_{1}^{\prime} \mathrm{I}^{\prime} \mathrm{G}^{\prime \prime} \mathrm{F} 18 / \mathrm{E}_{3}^{\prime} \mathrm{G}^{\prime} \mathrm{I}^{\prime} \mathrm{I}^{\prime \prime}\end{array}$ & $\begin{array}{l}\mathbf{O}_{1}(\mathbf{Y}) \mathbf{E}_{3}^{\prime} \mathbf{G}^{\prime}(\mathbf{I}) \mathbf{K}^{\prime} \mathbf{G}^{\prime \prime} \\
\mathbf{E}_{1}^{\prime} \mathbf{I}^{\prime} \mathbf{G}^{\prime \prime} \mathbf{F 1 8}\end{array}$ & - \\
\hline \multicolumn{5}{|c|}{ Normande } \\
\hline 50 & F & $\mathrm{G}_{1} \mathrm{O}^{\prime} \mathrm{P}_{1}^{\prime} \mathrm{A}^{\prime \prime} \mathrm{B}^{\prime \prime} \mathrm{I}^{\prime \prime} / \mathrm{I}_{1} \mathrm{QD}^{\prime} \mathrm{E}_{1}^{\prime} \mathrm{G}^{\prime \prime} \mathrm{F} 18$ & $\begin{array}{l}\left(G_{1}\right) \mathbf{Q D} \mathbf{E}_{3}^{\prime} \mathbf{O}^{\prime}\left(\mathrm{A}^{\prime \prime}\right) \\
\mathbf{G}^{\prime \prime}\left(\mathrm{I}^{\prime \prime}\right) \mathbf{F 1 8}\end{array}$ & - \\
\hline \multicolumn{5}{|c|}{$\begin{array}{c}\text { Française } \\
\text { Frisonne } \\
\text { Pie-Noire }\end{array}$} \\
\hline 51 & $\mathrm{~F}$ & $\mathrm{BG}_{2} \mathrm{KYA}^{\prime} \mathrm{O}^{\prime} \mathrm{I}^{\prime \prime} \mathrm{F} 4 / \mathrm{BO}_{1} \mathrm{YD}^{\prime} \mathrm{I}^{\prime \prime}$ & BYD'O'I'I & - \\
\hline
\end{tabular}

Ces cas sont numérotés à la suite de ceux répertoriés précédemment (Grosclaude et al., 1979). (1) Se réfère au parent ayant produit le gamète recombiné. (2) Facteur entre parenthèses : aucune conclusion n'est possible pour ce facteur car le même facteur fait partie du phénogroupe reçu de l'autre parent; en caractère gras : phénogroupe n'ayant encore jamais été observé dans la race. (3) + : La transmission à la génération suivante du phénogroupe recombiné a pu être observée au moins une fois.

These cases are numbered following those previously listed (GRosclaude et al., 1979). (1) Refers to the parent that produced the recombined gamete. (2) Factor in brackets : no conclusion can be drawn, since this factor is present in the phenogroup received from the other parent; in bold type : a phenogroup that was never observed before in the breed. (3) $+:$ Transmission of the recombinant phenogroup to the next generation was observed in the blood type of at least one offspring. 


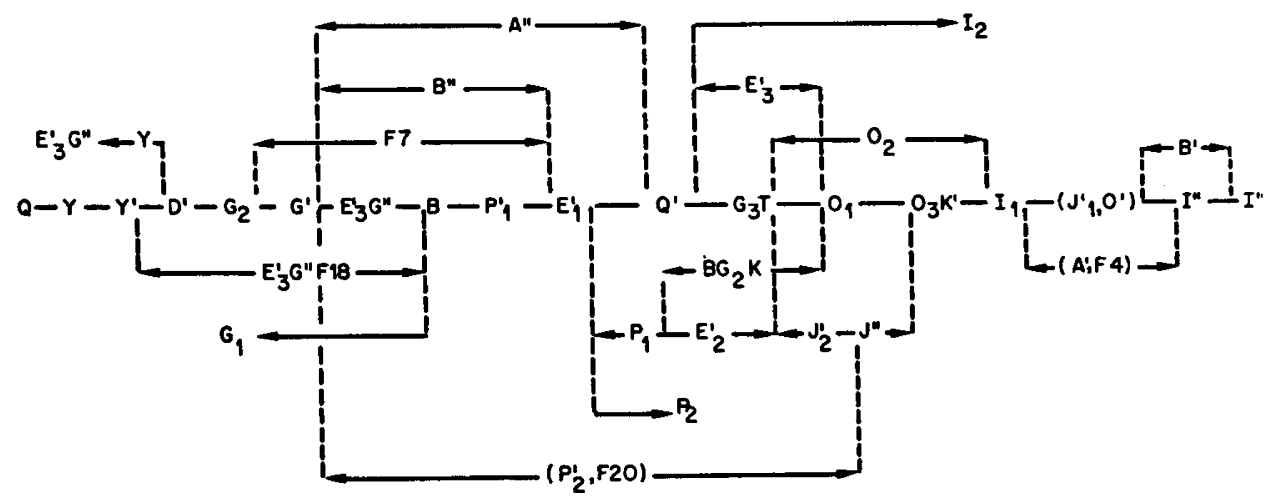

FIG. 1

Etat actuel de la carte génétique du système de groupes sanguins $B$ des bovins.

Present status of the genetic map of the B system of cattle blood groups.

La séquence de 18 déterminants génétiques, ou groupes de déterminants génétiques, est donnée dans la ligne médiane. Les autres déterminants génétiques identifiés sont localisés selon les flèches : $\leftrightarrow$, définit la région dans laquelle se trouve un déterminant particulier (par exemple le déterminant de $A^{\prime \prime}$ se trouve entre ceux de $G^{\prime}$ et de $\left.Q^{\prime}\right) ; \leftarrow($ ou $\rightarrow$ ), indique qu'un déterminant est localisé à gauche (ou à droite) d'un autre déterminant (par exemple le déterminant de $G_{1}$ est situé à gauche de celui de $B$ ). Déterminants entre parenthèses : leur ordre n'a pu être établi. Les groupes de facteurs non inclus dans des parenthèses $\left(E_{3}^{\prime} G^{\prime \prime}, E_{3}^{\prime} G^{\prime \prime} F 18, G_{3} T, O_{3} K^{\prime}, B_{2} K\right)$ correspondent à des associations très étroites.

The sequence of eighteen genetic determinants, or groups of genetic determinants, is given in the median line. The other genetic determinants shown are located according to the arrows : $\leftrightarrow$, defines the region in which a particular determinant occurs (e.g. the determinant of $A^{\prime \prime}$ occurs between those of $G^{\prime}$ and $\left.Q^{\prime}\right) ; \leftarrow($ or $\rightarrow)$, indicates that this genetic determinant is located to the left (or the right) of another determinant (e.g. the determinant of $G_{1}$ is to the left of that of $\left.B\right)$. Determinants in brackets : their order could not be established. Groups of factors not enclosed in brackets $\left(E_{3}^{\prime} G^{\prime \prime}, E^{\prime} G^{\prime \prime} F 18, G_{3} T\right.$, $\left.\mathrm{O}_{3} \mathrm{~K}^{\prime}, B \mathrm{G}_{2} \mathrm{~K}\right)$ correspond to very close associations.

Par rapport à la version proposée antérieurement (figure 3 dans Grosclaude et al., 1979), les conclusions qui peuvent être tirées de l'examen des 11 cas répertoriés dans le tableau 1 permettent d'introduire, dans la carte génétique du système $\mathbf{B}$ (fig. 1) les nouveautés suivantes :

- Le déterminant génétique du facteur $O_{1}$ se trouve désormais localisé «à la droite » de celui du facteur $T$. On notera, sur ce point, la concordance des observations déductibles des cas $n^{\circ} 44$ et 48 . Ceci permet d'insérer le déterminant du facteur $O_{1}$ dans. la séquence médiane de la carte du système.

- En ce qui concerne cette séquence médiane, la position relative du déterminant du facteur $B$, et de ceux des facteurs $P^{\prime}{ }_{1}$ et $Q^{\prime}$, qui faisait partie des déductions de l'analyse d'une famille de phénogroupes pouvant dériver du phénogroupe $\mathrm{BO}_{3} \mathrm{YA}^{\prime} \mathrm{E}_{3}^{\prime} \mathrm{G}^{\prime} \mathrm{P}^{\prime}{ }_{1} \mathrm{Q}^{\prime} \mathrm{G}^{\prime \prime} \mathrm{I}^{\prime \prime}$ (fig. 4 dans Grosclaude et al., 1979), est confirmée par le cas $n^{\circ} 45$. 
- Le déterminant génétique du facteur $\mathrm{E}_{1}^{\prime}$ est localisé avec plus de précision, entre ceux des facteurs $\mathrm{P}_{1}^{\prime}$ et $\mathrm{Q}^{\prime}$ (cas $\mathrm{n}^{\circ} 43$ et 50 ). Dans ces conditions, le facteur $\mathrm{E}^{\prime}{ }_{1}$ a été introduit dans la séquence médiane de la carte du système à la place du facteur $E_{2}^{\prime}$, dont le déterminant avait été localisé à gauche de celui de $\mathrm{Q}^{\prime}$, au lieu de $\mathrm{J}_{2}^{\prime}$, à la suite d'une erreur d'identification d'un phénogroupe.

- Les facteurs $\mathrm{E}_{1}^{\prime}$ et $\mathrm{E}_{2}^{\prime}$ sont contrôlés par des déterminants génétiques distincts, séparés par au moins un autre déterminant, celui de $P_{1}$ (cas $n^{\circ} 43$ et 47 ); $E_{2}^{\prime}$ a donc été placé entre $P_{1}$ et $J_{2}^{\prime}$, en faisant l'hypothèse que le facteur $E_{2}^{\prime}$ du phénogroupe $\mathrm{BG}_{2} \mathrm{KE}_{2}{ }_{2} \mathrm{O}^{\prime} \mathrm{A}^{\prime \prime}$ est le même que celui du phénogroupe $\mathrm{O}_{3} \mathrm{E}_{2}^{\prime} \mathrm{J}_{2}{ }_{2} \mathrm{I}^{\prime \prime} \mathrm{J}^{\prime \prime}$.

- Les bornes de l'intervalle dans lequel sont localisés les déterminants des facteurs $P_{2}$ (cas $n^{\circ} 49$ ), $P_{2}^{\prime}$ et $F 20$ (cas $n^{\circ} 42$ ), et surtout ceux du facteur $P_{1}$ (cas $n^{\circ} 41$, 42 et 47 ) et du groupe $\mathrm{BG}_{2} \mathrm{~K}$ (cas $\mathrm{n}^{\circ} 47$ et 51 ) sont désormais plus rapprochées. Il se confirme en particulier que les déterminants de $T$ et de $B_{2} K$ sont très voisins, comme l'avaient suggéré STORMONT (1972) et Grosclaude et al. (1979).

- Les déterminants des facteurs $A^{\prime \prime}$ (cas $n^{\circ} 42$ et 43 ) et $B^{\prime \prime}$ (cas $n^{\circ} 41$ et 50), qui n'avaient pas été pris en compte dans la version précédente de la carte génétique du système, ont été introduits dans la figure 1 , où ils sont d'ailleurs localisés dans la même zone.

Le cas $n^{\circ} 46$ mérite une attention particulière. En effet, on constate ici l'absence, dans le phénogroupe remanié, du groupe $E_{3}^{\prime} G^{\prime \prime}$, pourtant présent dans les deux phénogroupes parentaux. Or ce cas équivaut à une répétition du «cas LASSO * (GrosCLAUDE et al., 1979), dans lequel le phénogroupe recombiné, $\mathrm{O}_{\mathrm{x}} \mathrm{TB}^{\prime} \mathrm{G}^{\prime} \mathrm{O}^{\prime}$, dérivait des phénogroupes $\mathrm{BO}_{3} \mathrm{YA}^{\prime} \mathrm{E}_{3}^{\prime} \mathrm{G}^{\prime} \mathrm{P}_{1}^{\prime} \mathrm{Q}^{\prime} \mathrm{G}^{\prime \prime} \mathrm{I}^{\prime \prime}$ et $\mathrm{O}_{\mathbf{x}} \mathrm{TB}^{\prime} \mathrm{E}_{3}^{\prime} \mathrm{G}^{\prime} \mathrm{O}^{\prime} \mathrm{G}^{\prime \prime} \quad\left(\mathrm{BO}_{3} \mathrm{YA}^{\prime} \mathrm{E}_{3}^{\prime} \mathrm{G}^{\prime} \mathrm{P}_{1}^{\prime} \mathrm{Q}^{\prime} \mathrm{G}^{\prime \prime} \mathrm{I}^{\prime \prime}\right.$ étant donc commun aux deux cas). Comme la survenue de deux événements en principe rares, comme le double crossing-over ou la délétion, est moins vraisemblable que celle de deux crossing-over simples, on est donc conduit à admettre qu'il y a duplication du déterminant du groupe $E_{3}^{\prime} G^{\prime \prime}$, le premier site étant localisé entre ceux de $Y$ et de B (Grosclaude et al., 1979), le second « à gauche » de Y.

Mais cette conclusion conduit, en corollaire, à reconsidérer la position du premier site qui avait été placé «à gauche» de celui de $\mathrm{G}^{\prime}$ sur la base des observations faites sur un phénogroupe remanié dérivé précisément de $O_{x} T B^{\prime} E_{3}^{\prime} G^{\prime} O^{\prime} G^{\prime \prime}$ (cas $n^{0} 26$ ), qui se rapportent donc, en fait, à ce qui est désormais considéré comme le second site. Dans ces conditions, il paraît judicieux, à ce stade, de prendre en compte le cas décrit par Stormont \& SuzUKi (1962), cas qui démontre que dans le phénogroupe $Y_{1} E_{3}^{\prime} G^{\prime} Y^{\prime} G^{\prime \prime}$ présent en race Holstein (noté $\mathrm{O}_{\mathrm{x}} \mathrm{Y}_{1} \mathrm{E}_{3}^{\prime} \mathrm{G}^{\prime} \mathrm{Y}^{\prime}$ à l'époque), le déterminant génétique du facteur $E_{3}^{\prime}$ est situé «à droite » de celui de $G^{\prime}$. Le facteur $G^{\prime}$ et le groupe $E_{3}^{\prime} G^{\prime \prime}$ ont donc été intervertis dans la carte du système B (fig. 1). Finalement, nous avons également tenu compte, dans la figure 1, d'un cas de recombinaison observé par STORMONT (1981) chez un animal de race Tarentaise de son propre troupeau, qui montre que dans le groupe $\mathrm{YY}^{\prime}$, le facteur $\mathrm{Y}$ est « à gauche» du facteur $\mathrm{Y}^{\prime}$.

A noter que la carte représentée dans la figure 1 ne représente que la disposition relative des déterminants génétiques du système, et ne comporte aucune information sur leur distance relative. 


\section{B. Relation entre le taux apparent de recombinaison intra-race et le taux de recombinaison entre les bornes du système}

On voit facilement que, dans le cas d'un génotype homozygote au système $\mathbf{B}$, ou dans celui d'un génotype hétérozygote pour un seul déterminant génétique, le crossingover simple ne peut pas produire de phénogroupes remaniés et n'a donc pas d'effet décelable. D'autre part, pour tout autre génotype, le taux d'apparition de phénogroupes remaniés dépend de la distance entre les «bornes hétérozygotes du génotype 》 (les deux déterminants, à l'état hétérozygote, qui sont les plus espacés l'un de l'autre dans ce génotype). Le taux moyen d'apparition de phénogroupes remaniés dans une race, ou «taux apparent de recombinaison intra-race» est donc inférieur au taux de recombinaison entre les bornes du système $\left(\mathrm{d}_{\mathrm{QI}^{\prime}}\right)$.

Compte tenu des difficultés de mesure de la valeur effective du taux de recombinaison apparent intra-race (voir sur ce point la discussion), nous nous sommes attachés à approcher l'ordre de grandeur de ce taux par un calcul a priori qui, faute de données précises sur les distances entre les déterminants génétiques du système, requiert la formulation d'hypothèses sur ces distances :

Soient :

$f_{i}$ et $f_{j}$ les fréquences de deux des $n$ phénogroupes connus dans une race, $P_{i}$ et $P_{j}$, $\delta_{\left(P_{i} \cup P_{j}\right)}$ la distance entre les bornes hétérozygotes du génotype $B_{i} / B P_{j}$.

Le taux apparent de recombinaison intra-race, $\mathbf{R}$ est alors :

$$
R=2 \sum_{i=1}^{n-1} \sum_{j=i+1}^{n} f_{i} f_{j} \delta_{\left(P_{i} \cup P_{j}\right)} .
$$

Les hypothèses faites sur les distances entre les déterminants génétiques du système, qui permettent, pour chaque génotype, de calculer la valeur de $\delta$, sont indiquées graphiquement dans le modèle représenté dans la figure 2 . Ce modèle, dans lequel, par souci de simplification les déterminants génétiques du système $B$ sont regroupés en 15 sites distincts seulement, tient compte du petit nombre de données objectives dont on dispose, notamment sur la position relative des déterminants de $\mathrm{I}_{1}, \mathrm{~K}^{\prime}$ et $\mathrm{I}^{\prime \prime}$ (Grosclaude et al., 1979). Il intègre par ailleurs des observations bien recoupées, mais qui n'ont pas encore été quantifiées : la liaison étroite entre les déterminants des facteurs compris entre $D^{\prime}$ et $P^{\prime}{ }_{1}$ et la liaison étroite entre les déterminants des facteurs compris entre $\mathrm{T}$ et $\left(\mathrm{J}_{1}^{\prime}, \mathrm{O}^{\prime}\right)$, ainsi que le relatif espacement des facteurs $Q, Y$ et $\mathrm{Y}^{\prime}$ dans la partie " gauche 》 de la carte, $I^{\prime \prime}$ et surtout $I^{\prime}$ dans la partie «droite» (Grosclaude et al., 1979).

Les calculs effectués sur la base de ce modèle, pour 3 races (Charolaise, Montbéliarde, Normande), connaissant la fréquence des phénogroupes dans ces races (GrosCLAUDE, non publié) donnent, pour $\mathrm{R}$, les valeurs suivantes : $0,56 \mathrm{~d}_{\mathrm{QI}^{\prime}}$, en race Montbéliarde, $0,52 \mathrm{~d}_{\mathrm{QI}^{\prime}}$, en race Charolaise, et $0,45 \mathrm{~d}_{\mathrm{QI}^{\prime}}$ en race Normande. La valeur plus faible observée en race Normande s'explique, entre autres, par la faible fréquence du facteur $\mathrm{I}^{\prime}$ dans cette race (003, contre 0,34 en Montbéliarde et 0,16 en Charolaise), les écarts entre les fréquences des autres facteurs terminaux, $I^{\prime \prime}$ d'une part, $Q, Y$ et $Y^{\prime}$ de l'autre, étant moins accusés. A noter, par comparaison, qu'un modèle comportant la même disposition relative, des mêmes groupes de facteurs, mais en des sites équidistants, donnerait, pour $\mathrm{R}$, les valeurs suivantes : $0,68 \mathrm{~d}_{\mathrm{QI}^{\prime}}$, pour la race Charolaise, $0,60 \mathrm{~d}_{\mathrm{QI}^{\prime}}$ pour la Montbéliarde, et $0,66 \mathrm{~d}_{\mathrm{QI}^{\prime}}$ pour la Normande. 


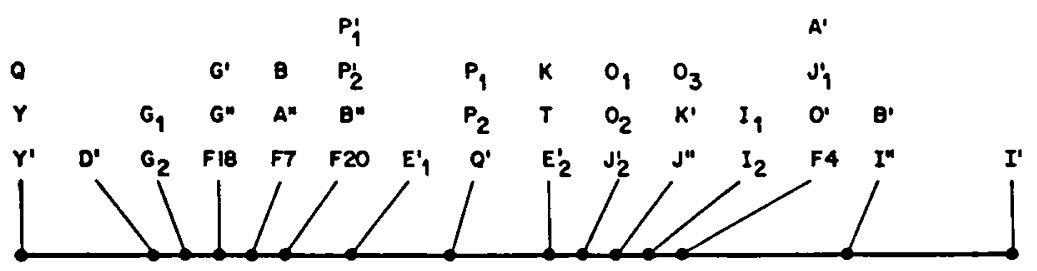

Fig. 2

Modèle de structure génétique du système $B$ retenu pour un calcul de taux de recombinaison apparent intra-race.

Model for the genetic structure of the $B$ system used for a calculation of the apparent within breed rate of recombination.

Les déterminants génétiques du système sont regroupés en quinze sites, la distance entre deux sites contigus étant un multiple (de 1 à 5 ) du $1 / 30^{\circ}$ de la longueur opérationnelle du segment d'ADN codant pour le système.

The genetic determinants of the system are grouped in fifteen sites, the distance between 2 contiguous sites being a multiple (1 to 5 times) of the 1/30th of the operational length of the DNA sequence coding for the system.

En retenant, pour $\mathrm{d}_{Q^{\prime}}$, la valeur de 0,7 p. 100, proposée antérieurement (GRosClaude et al., 1979), lordre de grandeur de $\mathrm{R}$, sur ka base du modèle proposé dans la figure 2 , est donc de 0,4 p. 100 .

\section{Discussion}

Nous avons démontré précédemment (Grosclaude et al., 1979), avec l'analyse du cas «VIOLON》, que des événements génétiques autres que le crossing-over simple, tels que le crossing-over double ou la délétion, pouvaient survenir dans un système de groupes sanguins complexe comme le système B. Dans le même travail, un autre cas, le cas «LASSO », faisait l'objet d'une discussion particulière : on observait en effet, dans ce cas, chez un descendant d'un taureau dont les deux phénogroupes comportaient le groupe de facteurs $E_{3}^{\prime} G^{\prime \prime}$, l'apparition d'un phénogroupe dépourvu de ces facteurs. Ce remaniement paraissait pouvoir être imputé à une délétion. Toutefois, l'intervention d'un crossing-over simple, qui impliquait l'existence d'un second déterminant génétique pour $E_{3}^{\prime} G^{\prime \prime}$, «à gauche » de celui de $Y$, n'était pas totalement rejetée.

La mise en évidence du cas $n^{\circ} 46$ (tabl. 1), qui équivaut à une répétition du cas *LASSO », oblige à reconsidérer le choix fait entre les deux interprétations. Il est logique d'attribuer désormais plus de vraisemblance à la survenue de deux cas de crossing-over simple, ce qui implique qu'il existe, "à gauche » du déterminant de $\mathrm{Y}$, un second déterminant pour $\mathbf{E}_{3}^{\prime} \mathrm{G}^{\prime \prime}$. Cette conclusion conduit, nous l'avons vu, à reconsidérer également la localisation du premier déterminant. Tout ceci montre, à l'évidence, les difficultés, voire les pièges rencontrés dans l'établissement de la carte du système $\mathrm{B}$. $\mathrm{Si}$ l'existence des deux sites pour $E_{3}^{\prime} G^{\prime \prime}$ devait être confirmée par la suite, il conviendrait 
alors, au cours de l'analyse génétique du système $\mathbf{B}$, d'identifier, pour chaque phénogroupe comportant $\mathrm{E}_{3}^{\prime} \mathrm{G}^{\prime \prime}$, le site impliqué, rien n'excluant d'ailleurs que certains phénogroupes puissent comporter deux fois ce groupe de facteurs.

Quoique Stormont (1981) ait pu démontrer, par l'observation d'un cas de recombinaison, que les déterminants génétiques des facteurs $\mathrm{Y}$ et $\mathrm{Y}^{\prime}$ étaient dissociables, il nous a paru préférable de maintenir, dans la carte du système, une distinction entre un site correspondant au déterminant génétique du facteur $\mathrm{Y}$ dans le groupe $\mathrm{Y}-\mathrm{Y}^{\prime}$, et un second site correspondant au déterminant de $Y$ seul. D'ailleurs, l'existence des sousgroupes $Y_{1}$ et $Y_{2}$ (non discriminés avec nos réactifs; $Y$ correspond à $Y_{2}$ ), conduit aussi à supposer qu'il existe plus d'un déterminant génétique pour les facteurs de spécificité $Y$. En ce qui concerne le site du second déterminant de $E_{3}^{\prime} G^{\prime \prime}$ (au moins présent dans les phénogroupes $\mathrm{O}_{x} \mathrm{~TB}^{\prime} \mathrm{E}_{3}^{\prime} \mathrm{G}^{\prime} \mathrm{O}^{\prime} \mathrm{G}^{\prime \prime}$ et $\left.\mathrm{A}^{\prime} \mathrm{E}_{3}^{\prime} \mathrm{G}^{\prime} \mathrm{G}^{\prime \prime} \mathrm{F} 4\right)$ il pourrait être localisé «à droite » de celui de $Q$ : il existe en effet, en race Charolaise, un phénogroupe $\mathrm{QA}^{\prime} \mathrm{E}_{3}^{\prime} \mathrm{G}^{\prime} \mathrm{G}^{\prime \prime} \mathrm{F} 4$ qui paraît dériver de $\mathrm{A}^{\prime} \mathrm{E}_{3}^{\prime} \mathrm{G}^{\prime} \mathrm{G}^{\prime \prime} \mathrm{F} 4$ par la simple addition de $\mathrm{Q}$ 《à gauche». De manière générale, il est clair que la partie de la carte du système $B$ située «à gauche» du déterminant de $\mathbf{B}$ comporte encore bien des imprécisions. Par contre, le reste de la carte paraît dans l'ensemble plus solidement établi, en particulier pour ce qui concerne la zone comprise entre les déterminants de $\mathrm{B}$ et de $\mathrm{O}_{3} \mathrm{~K}^{\prime}$, sur laquelle des précisions importantes ont été apportées dans le présent travail.

Le pourcentage de filiations erronées est nettement plus élevé, dans les races bovines, que celui des filiations dans lesquelles apparaissent, par recombinaison, un phénogroupe remanié. Cet état de fait est l'un des facteurs qui contribuent à rendre délicate la mesure directe du taux apparent de recombinaison intra-race, puisque l'incertitude ne peut pas toujours être levée sur l'interprétation à donner à des discordances entre les phénogroupes d'un produit, et ceux de ses parents; d'autre part cette mesure est compliquée par le fait que, dans une partie des filiations, le phénogroupe remanié par recombinaison est masqué, chez le sujet porteur, par son second phénogroupe. Ces difficultés expliquent l'absence d'études et de résultats fiables sur le taux apparent de recombinaison intra-race.

Le modèle de structure génétique du système $B$ retenu pour tenter d'approcher ła valeur de ce taux dans 3 races françaises appartient à la classe des modèles plausibles, et présente d'ailleurs une certaine ressemblance avec le modèle de RuITERKAMP et al. (1977). Toutefois il est à noter que nos résultats (Grosclaude et al., 1979, et ce travail) contredisent une partie des bases retenues par ces auteurs pour l'élaboration de leur modèle, notamment pour ce qui touche à l'idée que les facteurs sérologiquement apparentés à $\mathbf{E}_{4}^{\prime}$ seraient contrôlés par un groupe de déterminants génétiques étroitement liés ( $\mathrm{E}^{\prime}{ }_{4}$ cluster 》 dans la publication, en anglais, de Ruiterkamp et al.).

En tout état de cause l'analyse préliminaire effectuée dans le présent travail a pour but de poser les termes d'un problème qui n'avait pas, à notre connaissance, été abordé jusqu'ici. Des données plus complètes sur les distances entre les différents déterminants génétiques du système permettraient une estimation plus précise du taux apparent de recombinaison intra-race. Mais la collecte de ces données reste, dans l'espèce bovine, une entreprise de longue haleine.

Reçu pour publication le 12 octobre 1982. Accepté pour publication le 22 novembre 1982.

Note : Une erreur typographique est à corriger dans Grosclaude et al., 1979, tableau 1 : dans le cas $n^{\circ} 8$, le phénogroupe recombiné est $I_{1} O_{3} Q^{\prime} A^{\prime} E_{1}^{\prime} K^{\prime} Q^{\prime} I T^{\prime \prime} F 4$ (et non $I_{2} O_{3} Q^{\prime} A^{\prime} E_{1}^{\prime} K^{\prime} Q^{\prime} I^{\prime \prime} F 4$ ). 


\section{Références bibliographiques}

Bouw J., Fionentini A., 1970. Structure of loci controlling complex blood group systems in cattle. Proc. 11th Eur. Conf. Anim. Blood Grps (Warsaw, 1968), 109-113.

Carenzi C., Fiorentini G., Rognoni G., Bouw J., 1972. Misleading reagents for cattle bloodtyping. Proc. 12th Eur. Conf. Anim. Blood Grps (Budapest, 1970), 97-101.

Green P., 1966. Towards a tentative map of the genetic determinants of the B blood group system of cattle. Immunogenet. Lett., 4, 188-191.

Grosclaude F., Guerin G., Houlier G., 1979. The genetic map of the B system of cattle blood groups as observed in French breeds. Anim. Blood Grps biochem. Genet., 10, 199-218.

Oosterlee C.C., Bouw J., 1974. Loci structure in animal blood groups. Proc. 1st World Cong. Genet. applied to Livestock Prod. (Madrid), 1, 243-252.

ReNDEL J., 1958. Studies of cattle blood groups. IV - The frequency of blood group genes in Swedish cattle breeds, with special reference to breed structure. Acta Agric. scand., 8, 191-215.

Ruiterkamp W.A., SpeK C.M., Bouw J., 1977. Gene clusters in the blood group system B of cattle. Anim. Blood Grps biochem. Genet., 8, 231-240.

Stormont C., 1955. Linked genes, pseudoalleles and blood groups. Am. Nat., 89, 105-116.

Stormont C., 1972. The language of phenogroups. Haematologia, 6, 73-79.

Stormont C., 1981. A note on linear subtyping relationships. Papers dedicated to Prof. J. Moustgaard on the occasion of his 70th birthday, 190-193, Aio-Tyrk as, Odense, Denmark.

Stormont C., OWEN R.D., IRWin M.R., 1951. The B and C systems of bovine blood groups. Genetics, 36, 134-161.

Stormont C., Suzuki Y., 1962. A possible duplication of the B locus in cattle. Immunogenet. Lett., 2, 80-81. 